Artículos

\title{
EXHIBICIÓN Y PROGRAMACIÓN CINEMATOGRÁFICA EN LEÓN, MÉXICO, DESDE LA PERSPECTIVA DE LA NUEVA HISTORIA DEL CINE (1940 A 1970)
}

\author{
Jaime Miguel González Chávez \\ Universidad De La Salle, Bajío, México \\ Efraín Delgado Rivera \\ Universidad De La Salle, Bajío, México \\ Joel Ortega Contreras \\ Universidad Incarnate Word, Bajío, México \\ Philippe Meers \\ University of Antwerp, Bélgica \\ Autor para correspondencia: Jaime Miguel González Chávez, email: \\ jaimegonzalezchavez@gmail.com
}

\section{Resumen}

Esta investigación forma parte del enfoque New Cinema History (Maltby, Biltereyst y Meers, 2011). Describe la programación y exhibición cinematográfica entre los años 40 y 70 en León, uno de los 10 centros urbanos más importantes de México. Metodológicamente es una réplica del proyecto original de Flandes (Biltereyst y Meers, 2007). La investigación se apoya en entrevistas a profundidad, trabajo de archivo y de carteleras. Se ofrece un análisis del papel social de la cultura cinematográfica como resultado de tensiones entre fuerzas comerciales e ideológicas, en interacción con los procesos de urbanización y modernidad. En el caso de León, el consumo de cine nacional y extranjero muestra una correlación en las audiencias de acuerdo con la brecha económica, sobre todo durante el proceso de transformación de la ciudad rural a urbana.

Palabras clave: new cinema history, historia social del cine en México, cine en León, exhibición y programación cinematográfica.

\begin{abstract}
This research is part of the New Cinema History approach (Maltby, Biltereyst y Meers, 2011). It describes the programming and cinematographic showing between the 40's and the 70's in León, one of the 10 most important urban centers in Mexico. Methodologically it is a replica of the original Flanders project (Biltereyst y Meers, 2007). The research is supported by indepth interviews, archival work and billboards. It offers an analysis of the social role of the
\end{abstract}


cinematographic culture as a result of tensions between commercial and ideological forces, interacting with the processes of urbanization and modernity. In León's case, the consumption of national and foreign films shows a correlation in the audiences according to the economic gap, especially during the transformation process from rural to urban city.

Keywords: new cinema history, social history of cinema in Mexico, cinema in León, exhibition and cinematographic programming.

Recibido:18/02/2019

Aceptado:28/06/2019

\section{Introducción}

El abordaje de la historia social del cine New Cinema History- plantea un nuevo enfoque de estudio el cual observa las prácticas socioculturales que acompañan y derivan de los procesos de producción, distribución y consumo cinematográfico para explorar su vínculo al desarrollo de la modernidad y el urbanismo en la ciudad, la aparición de los soportes materiales de esta expresión cultural, así como las experiencias de asistencia al cine. Es una propuesta relativamente nueva que atiende estos procesos habitualmente ignorados por los estudios especializados en cine (Maltby, Biltereyst y Meers, 2011).

Esta perspectiva facilita el análisis de procesos sociales para entender las instituciones mediáticas a partir de tres puntos de entrada (Mosco, 2006).

1. Mercantilización: proceso mediante el cual se transforma el uso para intercambiar valores.
2. Especialización: transformación del espacio con el tiempo, o el proceso de extensión institucional.

3. Estructuración: construir estructuras como resultado de la acción social.

A partir de este enfoque se aborda la trayectoria de la industria cinematográfica en la ciudad de León, Guanajuato, México, en un periodo que comprende los años que van de 1940 a 1979. El estudio de caso explora la exhibición y programación en esta ciudad, tema muy poco explorado en el país con excepción de unos cuantos trabajos sobre la ciudad de México (Mantecón, 2000; Repoll, Portillo y Meers, 2014) y Monterrey (Lozano, Biltereys, Frankenberg, Meers e Hinojosa, 2012).

Este recorte temporal es particularmente interesante ya que corresponde al periodo de transformación profunda de la sociedad mexicana, que incorporaba los procesos de modernización y de urbanización en ciudades de provincia, como es el caso de León. En segunda 
instancia, porque corresponde al auge y decadencia de la producción cinematográfica mexicana en su reconocida Época de Oro.

En términos económicos, la historia moderna de la ciudad de León, es decir, de la época posterior a las guerras mundiales y hasta finales del siglo XX, muestra la consolidación de una fuerte industria de la piel y particularmente del calzado. ${ }^{1}$ Sin embargo, los cimientos del desarrollo de la ciudad como centro urbano pueden ubicarse a partir de 1940, cuando ocurre la transformación de región rural a urbana (García Gómez, 2010), cuando calles empedradas, drenaje a cielo abierto, tranvías y poca movilidad motorizada eran parte de un ambiente que, poco a poco, incorporaba las modificaciones arquitectónicas premodernas que culminan en la década de los años setenta.

En 1940, con 103,105 habitantes, se inicia una intervención urbanística que transformaría la ciudad. Esto generó un despliegue de servicios, intervenciones urbanísticas y tecnológicas asociados al crecimiento económico y poblacional. Las diferentes formas de habitar la ciudad se transformaban; la industria del ocio y el espectáculo no sería la excepción.

Es en esta fase de profundos cambios en las formas de vida de los habitantes de León que exploramos el papel de una industria como la del cine, la cual, en su dinámica, se asoció a esta impronta tecnológica que caracterizó el sueño de modernidad de las naciones en desarrollo. Una compleja industria cultural, que asocia el trabajo de distintas cadenas de intercambio económico.

Para generar la información base del presente estudio, se aplicó la metodología comparativa diseñada para la red de estudios de historia social del cine (Biltereyst y Meers, 2016; Allen, 2006). Este diseño permite construir una base de datos de acceso mundial, que constituye un reto abierto para la búsqueda de similitudes y diferencias analíticas entre diversas ciudades participantes alrededor del mundo. Los métodos aplicados fueron:

Tabla 1. Métodos de investigación aplicados

\begin{tabular}{ll}
\hline Método & Objetivo \\
\hline Cartografía de las salas de cine. & $\begin{array}{l}\text { Describir las transformaciones de la oferta de } \\
\text { salas de cine en la ciudad de León. }\end{array}$ \\
$\begin{array}{l}\text { Investigación documental de la cartelera } \\
\text { cinematográfica de la ciudad de León. } \\
\text { Analizar la oferta de películas en carteleras de } \\
\text { salas de cine de León. }\end{array}$ \\
$\begin{array}{l}\text { Historia de vida y entrevista a a } \\
\text { profundidad de especialistas y audiencias } \\
\text { del cine. }\end{array}$ & $\begin{array}{l}\text { Describir la experiencia fílmica de las } \\
\text { audiencias. }\end{array}$ \\
\hline
\end{tabular}
Fuente: Elaboración propia.

1 Es a partir de la década de 1940 que la economía de la ciudad centró sus actividades en la cadena productiva del cuero, calzado, proveeduría y marroquinería, haciéndose llamar la capital mundial del calzado. Actualmente, el proyecto de gobierno reorientó su economía a los servicios y la industria automotriz. 
La investigación documental también es fundamental, pero como se mencionó, existe una carencia de sistematización en este sentido, por lo que resulta pertinente y necesario ahondar proyectos que abonen hacia una memoria social de cine.

\section{Antecedentes: León y sus prácticas lúdicas previas a 1940}

Dentro de las actividades lúdicas de la preurbanidad leonesa -inicios del siglo XX hasta 1940 - María de la Cruz Labarthe (1995) identifica particularmente las actividades en espacios públicos, tales como el vuelo de papalote, paseos y días de campo a lo largo del río de los Gómez —al cual, cuentan, llegaban lavanderas y ciudadanos "con música, cantando unos por el camino, otros bailando bajo la sombra de los árboles y otros merendando tranquilamente sobre la verde alfombra del campo" (García Saavedra, 1985, pp. 1718)_; bailes y música en plazas públicas como las de Coecillo, San Miguel, Santiago o San Juan de Dios; además, había espectáculos taurinos en la Plaza de Toros (fundada en 1844 para albergar a 6,000 espectadores); espectáculos de óperas, sainetes y hasta can-can en la Plaza de Gallos; diversidad cultural ofertada en el teatro Manuel Doblado (el cual se convirtió en cine en el año 1916), así como veladas literarias, festejos, ferias, charrería y deporte.
En el caso del cine, su incorporación fue temprana. Tomó la forma de un espectáculo itinerante patrocinado por empresarios, muchas veces con motivos publicitarios. En el estado de Guanajuato fue impulsado por asociaciones y personajes como Manuel Isunza, Cinematógrafo Pathé, Rivera Corral y Cía., los Hnos. Becerril o promotores ligados al empresario francés Ernesto Pugibet como Eduardo M. Alva, José Francisco B. Moyano o Arturo Zarco del Valle (Leal y Barra, 2017).

Las primeras exhibiciones de 1897 se hicieron en un saloncillo que, con el nombre de La Concordia, se improvisó en el Portal Bravo. Allí, la firma de los Hermanos Becerril hizo correr las cintas cinematográficas en un aparato manual. Años más tarde, por 1905, la fábrica de cigarros El Buen Tono exhibió documentales en la plaza principal; no se quedó atrás la propaganda de la Tabacalera Mexicana con las proyecciones que ofreció en 1907 en la Plaza de Gallos. El primer salón de cine, por 1913, el Elite, se situó en la calle Honda. En 1916 el teatro Doblado fue acondicionado con butacas para hacer las veces de sala de cine, en 1914 se abrió el salón París y en 1920 el Olimpia (Labarthe, 1995, p. 10). 
Los años posrevolucionarios y la guerra cristera de 1926 a $1929^{2}$ constituyeron un impasse que ralentizó el desarrollo de actividades económicas y culturales de la ciudad, impactando la década de 1930.

Sin embargo, para los años cuarenta, la situación cambiaría. En 1939, Rafael Rangel, gobernador del estado de Guanajuato, anuncia "la recaudación más elevada de la historia del estado $(\$ 3,797,000.00)$, lo que representaba un superávit de más de $\$ 178,000.00$ sobre los egresos” (Guzmán López, 2014, p. 118). Es así que un renovado impulso a la modernidad de la urbe se instaura derivado de un aparente crecimiento económico, así como una gradual estabilidad en la sociedad leonesa.

\section{La ciudad premoderna y la cultura cinematográfica (1940-1970)}

El influjo de una modernidad posterior a las guerras mundiales influenció la vida económica y cultural en el país. Arrancando la cuarta década del siglo XX, México vive el llamado "milagro mexicano", 3 el cual impulsa la transformación de una nación

2 Esta guerra, también denominada Cristiada, confrontó, a peones, campesinos, presbíteros y religiosos que combatían al Gobierno y las políticas de Plutarco Elías Calles, quien promovía limitar la participación del clero en la política mexicana, desatando una insurrección en varias zonas del país, principalmente de Guanajuato, Jalisco, Querétaro y Zacatecas.

${ }^{3}$ El llamado Milagro Mexicano fue el nombre con el que se designó a la época de bonanza agrícola a una industrial de configuración urbana. México celebró el convenio de intercambio de mano de obra con Estados Unidos en 1942, el Programa Bracero, del cual Guanajuato fue un actor fundamental, teniendo a Irapuato como centro ferrocarrilero. "Durante los años de guerra (1942-1945), México aportó la mano de obra de 302,775 braceros, de los cuales el $13.6 \%$ fueron guanajuatenses, prevaleciendo sobre los de Jalisco (11.2\%), Chihuahua (10.7\%), Michoacán (10.6\%), Durango (9.4\%) y Zacatecas (9.3\%)" (Guzmán López, 2014, p. 122).

A partir de 1940 soplan vientos de progreso; León se consolida con una vocación industrial mediante el desarrollo de fábricas de textiles, de múltiples curtidurías $\mathrm{y}$, principalmente, con las fábricas de zapatos, las cuales veían un auge en su demanda por la inclusión de México en la Segunda Guerra Mundial.

La ciudad reconfiguró, como en toda sociedad en proceso de especialización técnica, los tiempos para el trabajo, el espacio para la religiosidad 94\% católica para el caso de León- y sus prácticas recreativas. Los tiempos laborales y de ocio también se especializaron ${ }^{4}$

económica impulsada por la Segunda Guerra Mundial, la cual tuvo inicio a partir de 1940.

4 "La invención del reloj mecánico en el siglo XVII abrió las puertas de una nueva dimensión temporal: el tiempo abstracto y lineal de la Modernidad. Los sujetos dejaron de seguir el ritmo de la naturaleza o el repicar de las campanas medievales para organizar sus vidas a partir del tic tac mecánico de las máquinas. De esta manera el tiempo pasó a ser un bien que podía ser consumido, fragmentado, comprado o 
consolidando una nueva práctica lúdica: la asistencia regular al cine.

En la década de 1940 se consolida en la ciudad una oferta de seis salas, con una programación de películas claramente diferenciada, derivada de una sociedad que mostraba una notoria brecha entre las clases obreras o populares y las clases acomodadas.

\section{La segmentación económica del}

consumo del cine de León en las décadas

\section{de los años cuarenta a setenta}

La ciudad de León muestra condiciones de desigualdad económica que contrastan con su bonanza; estas se observan hasta la actualidad en un municipio que, a pesar de considerarse como una de las diez economías más importantes de México, es también cuarto lugar en pobreza urbana. Una pobreza es visible también desde la perspectiva territorial donde aproximadamente $40 \%$ de la superficie de la zona urbana y $42 \%$ de los habitantes ahí establecidos eran, para 2010, pobres (González Chávez, 2016, p. 63; Instituto Municipal de Planeación Urbana, 2016).
Esta configuración se puede rastrear a 1940, según menciona García Gómez (2010) en su análisis de las Transformaciones Urbanas de León en el siglo $X X$. La ciudad, dice, muestra una segregación infraestructural que hoy se conoce bien, fruto de la aparición de colonias irregulares y periféricas. Es durante esta reconfiguración que las prácticas culturales muestran también una manifestación de ofertas diferenciadas y diferenciantes.

Como menciona Lewis (1958), desde 1895 a 1940 la clase media había crecido de $7.8 \%$ a $15.9 \%$, resultado de la industrialización y la concesión de salarios estables en el país; la ciudad de León no fue la excepción. Cuenta de esto es que el cine se convirtió en un espectáculo al que se podía acceder desde todas las clases sociales, pero siempre con la distinción que concedía la clase social.

Los cines integraron estas características. La audiencia identifica, según las entrevistas realizadas, una oferta de cines diferente entre la clase más acomodada y la clase popular. vendido. Esta concepción, nacida en la Europa moderna, se ha ido difundiendo en todas las sociedades - de la ciudad al campo, de Occidente a Oriente, del norte al sur-, las cuales dejaron progresivamente de mirar hacia el sol para fijar su atención en las manecillas de los relojes" (Scolari, 2008, p. 274). 
Tabla 2. Datos de salas de cine en León 1930-1950

\begin{tabular}{|c|c|c|c|c|c|}
\hline $\begin{array}{c}\text { Nombre de } \\
\text { sala }\end{array}$ & Calle y núm. & $\begin{array}{c}\text { Fecha } \\
\text { construcción }\end{array}$ & $\begin{array}{l}\text { Clase } \\
\text { social }\end{array}$ & $\begin{array}{l}\text { Precio } \\
\text { boleto }\end{array}$ & $\begin{array}{c}\text { Año } \\
\text { precio }\end{array}$ \\
\hline $\begin{array}{c}\text { Teatro } \\
\text { Manuel } \\
\text { Doblado }\end{array}$ & $\begin{array}{c}\text { Pedro Moreno } \\
202 \text { esquina con } \\
\text { Hermanos } \\
\text { Aldama, Centro } \\
\text { Histórico, } 37000 \\
\text { León, Gto. }\end{array}$ & 1880 & $\begin{array}{l}\text { Alta, } \\
\text { Media }\end{array}$ & Luneta $\$ 1$ & 1931 \\
\hline Teatro Vera & $\begin{array}{l}5 \text { de Mayo, sin } \\
\text { número, } 37000 \\
\text { León, Gto. }\end{array}$ & 1950 & $\begin{array}{l}\text { Alta, } \\
\text { Media }\end{array}$ & $\begin{array}{l}\text { Localidad } \\
\text { \$4, Luneta } \\
\$ 2.50, \\
\text { Niños } \$ 1\end{array}$ & 1940 \\
\hline Cine Isabel & $\begin{array}{l}\text { Artes 120, Col } \\
\text { de Santiago, } \\
37327 \text { León, } \\
\text { Gto. }\end{array}$ & 1930 & $\begin{array}{c}\text { Media, } \\
\text { Baja }\end{array}$ & Luneta $\$ 1$ & 1931 \\
\hline Cine Ideal & $\begin{array}{c}\text { Callejuela o } \\
\text { Pasaje Padilla, } \\
\text { Centro } \\
\text { Histórico, } 3700 \\
\text { León, Gto. }\end{array}$ & 1928 & Alta & $\begin{array}{l}\text { Localidad } \\
\quad \$ 4\end{array}$ & 1940 \\
\hline Cine Hernán & $\begin{array}{l}\text { Pino Suárez. } \\
\text { 203, Centro, } \\
\text { 37000 León, } \\
\text { Gto. }\end{array}$ & 1930 & $\begin{array}{l}\text { Alta, } \\
\text { Media }\end{array}$ & $\begin{array}{l}\text { Luneta } \$ 5, \\
\text { Balcón } \$ 3\end{array}$ & 1940 \\
\hline Cine Coliseo & $\begin{array}{c}\text { Reforma } 320, \\
\text { Centro, } 37000 \\
\text { León, Gto. }\end{array}$ & 1946 & $\begin{array}{c}\text { Media, } \\
\text { Baja }\end{array}$ & $\begin{array}{c}\text { Luneta } \\
\$ 3.50, \\
\text { Balcón \$2 }\end{array}$ & 1940 \\
\hline
\end{tabular}

Fuente: Elaboración propia, a partir de entrevistas a profundidad y archivo histórico

Esta diferenciación se manifiesta en el discurso, en una distancia social que, en el caso del cine, pareciera más de orden simbólico. No obstante, el costo de entrada también fue un factor diferenciador importante con un tercio de diferencia de costo promedio.

La distribución geográfica de las salas de cine fue mayoritariamente en la zona centro de León donde se aglomeraba gran cantidad de personas que realizaban sus actividades diarias de abasto y esparcimiento, además de la cercanía con centros de comercio de piel y calzado que también se ubicaban en esta misma zona o relativamente cerca.

Algunas personas, generalmente obreros de la industria cuero-calzado, preferían cines como el Cine Isabel; otros, de clase más acomodada, preferían el Cine Hernán:

$$
\begin{aligned}
& \text { en el Hernán íbamos las que } \\
& \text { llevábamos los zapatitos boleados } \\
& \text { y todo eso, y en el Isabel entraba } \\
& \text { toda la pelusa. Ahi entraba de todo, } \\
& \text { y como en ese tiempo de donde } \\
& \text { estaba se hacian filas de } \\
& \text { borrachitos tirados o mugrosos, }
\end{aligned}
$$


muy feo, mejor no ibamos al Cine Isabel [...] Además, el Cine Hernán, el Cine Américas y el Reforma eran salas muy limpias, mientras otros eran salas malolientes (Señora N., 77 años, comunicación personal, septiembre de 2017).

El Cine Hernán contaba con balcón y luneta con gradas de madera, mientras que en el Cine Isabel eran de cemento. En este último, además, se contaba con sección de galería.

La de cine Isabel estaba abajo la luneta. Era muy frio en tiempo de baja temperatura porque nada estaba acojinado, nada... y entraba el aire frio por donde quiera; para llegar al balcón eran dos vueltas de gradas; la galería estaba más alto, eran como tres o cuatro vueltas, era lo más barato, pero estaba bien alto en galería (Juan N., 75 años, comunicación personal, septiembre de 2017).

Además del mencionado Cine Hernán —ubicado en la calle 5 de mayo-, estaba el Cine Vera, sobre la misma avenida, pero en dirección norte. Un "lujoso" y "renovado" cine reconstruido por su dueño original, Guillermo Vera, después de que un gran incendio lo consumiera el 30 de junio de 1929. En cuanto a sus públicos, este recinto era frecuentado por la alta sociedad leonesa.

Por otra parte, en el Cine Isabel, en colindancia con el legendario barrio fundacional del Coecillo - hoy convertido en un amplio estacionamiento-, en la calle Artes del barrio de Santiago, acudía una audiencia con gran arraigo en las clases populares. Este cine contaba con promociones de $2 \times 1$ los viernes, en la que entraban dos personas con un mismo boleto, y podían ver tres películas por 10 centavos. Este cine contaba con dos tipos de localidades: luneta y galería. En él, como en todos los de este periodo, se programaba en mayor porcentaje cine mexicano. Pero estas no eran las únicas opciones para disfrutar de las películas de temporada a bajo costo.

El Cine Coliseo era también una opción para la clase obrera y popular, ubicado en las inmediaciones del centro de la ciudad, justo a un costado del Mercado Aldama —el mayor centro de abasto-, o mejor conocido como el mercado de la soledad en alusión a la Virgen de la Soledad, patrona de los comerciantes de la ciudad. En su cartelera predominaba la programación del cine mexicano con $64.3 \%$, mientras que el cine estadounidense alcanzaba únicamente $7.1 \%$, y el resto era de películas de diversos países (figura 2). Este cine, que no era otra cosa que una plaza de toros adaptada, contaba con "un amplio semicírculo de gradas y butacas sobre algo así como tierra apisonada que 
humedecían antes de cada función" (Cortés Gaviño, 1995, p.24).

También se realizaban proyecciones esporádicas en los barrios, como el Pinocho en el barrio de San Juan de Dios, en el Templo de la Soledad, el del Coecillo, el Juventud de la Parroquia del Sagrario, entre otros (Archivo Histórico de la Ciudad de León, 1998; citado en Ruel S.A. de C.V.).

Era común que las escuelas organizaran visitas de matiné a los cines para acercar a los alumnos a esta experiencia. Grupos escolares llenaban las salas de cine que entonces contaban con un gran número de butacas - a veces para más de mil personas - por lo que no era ningún problema acudir con los estudiantes. "Los niños aprovechaban los intermedios para correr hacia la tarima de la pantalla y corrían sobre ella, jugando y gritando, deslizándose desde la parte superior, mientras algunos padres de familia o acompañantes mayores surtían las palomitas de maíz y refrescos en la tienda del lugar" (Señora N., 77 años, comunicación personal, septiembre de 2017).

La época de los años sesenta fue un momento de desarrollo importante para el cine de la ciudad con la aparición de una mayor oferta de salas.

En la calle Pino Suárez, esquina con Díaz Mirón, o al día de hoy calle Reforma, estaba el Cine Reforma y el Cine Américas. Estos cines traían muchas películas importantes, tanto mexicanas como extranjeras. Ahi proyectaron Los Girasoles, de Rusia; eran películas de primer mundo. También El Santo contra las momias, películas de Rodolfo de Anda, el "Indio" Fernández, Pedro Infante, Jorge Negrete... Los cines que eran importantes en León eran el Cine Américas y el Cine Reforma, que estaban juntos, Cine Vera en la calle Pino Suárez, ubicado un poquito más adelante.

En el año 1965, más o menos, construyeron el Cinema Estrella, frente al Fiesta Americana, ahora Hotel Hotson. Un mega cine que para aquella época era de los más modernos; enorme, ya tenía nueva tecnología. En la calle Álvaro Obregón, a media cuadra de la Catedral, estaba el Cine León, que usaban también para eventos sociales, como la coronación de la Reina de la Ciudad. Posteriormente, en la calle Alud instalaron el Cine Insurgentes, ahora es un bodegón y una papelería en la parte baja. En esa época había muchos cines en León (Señor N., 71 años, comunicación personal, septiembre de 2017).

Cabe mencionar que las cuatro salas que estaban en funcionamiento en 1952, siguieron funcionando hasta entrada 
la década de los años setenta. Dos de ellas pertenecían a Guillermo Vera (el Cine Isabel $\mathrm{y}$ el Cine Vera), quien fuera presidente municipal a finales de los cuarenta y figura trascendente para la historia del cine en León y la región.

En el segundo lustro de la década de los cuarenta no solo administra y opera cines en León: tiene la concesión del mismísimo teatro Juárez en Guanajuato capital y tiene tres cines en Aguascalientes y otros tantos en Lagos de Moreno, San Francisco del Rincón, San Luis de la Paz, Dolores Hidalgo... y en 1948 inaugura su nuevo y lujoso cine Vera [...] un total de 18 cines integran su empresa Espectáculos Unidos (que incluye la lucha libre) (Alegre, 1995, pp. 21-22).

Por este motivo, de la misma manera que se observa con los precios, no existe una relación aparente entre una segmentación de públicos y la ubicación de las salas, pues compartían un conglomerado bastante diversificado. Sin embargo, como ya se mencionó, existe una distancia simbólica entre cada una de las ofertas las cuales segmentaban a sus públicos (tabla 2).

Los cines visitados por audiencias de clase acomodada y los cines para audiencias populares exhibieron películas y contenidos diferenciados en su cartelera. Para determinar este proceso, se trabajó con la estadística de películas programadas cada sábado desde enero de 1940 a diciembre de 1979 haciendo énfasis en el origen de la producción (nacional o extranjera).

Es importante acotar que no hay, dentro de las entrevistas, una diferenciación expresa entre las salas y sus públicos por nivel socioeconómico apreciativo a partir de la década de los sesenta, por lo que únicamente se ha utilizado este criterio hasta esta fecha. Es decir, a partir de la década de los sesenta, no se aplica esta distinción.

\section{La programación del cine en León en las décadas de 1940 a 1970}

Las diferentes audiencias que acudían a los cines de la ciudad, constituyeron también una presión en la demanda de los contenidos cinematográficos programados en las salas. Esta distinción es clara cuando se observan los consumos de películas extranjeras y nacionales.

En la estadística de proyecciones sabatinas, el comportamiento en cada década de acuerdo al origen de las películas (nacionales o extranjeras) es el siguiente: 
Figura 1. Porcentaje de programación según su origen de producción, nacional o extranjera

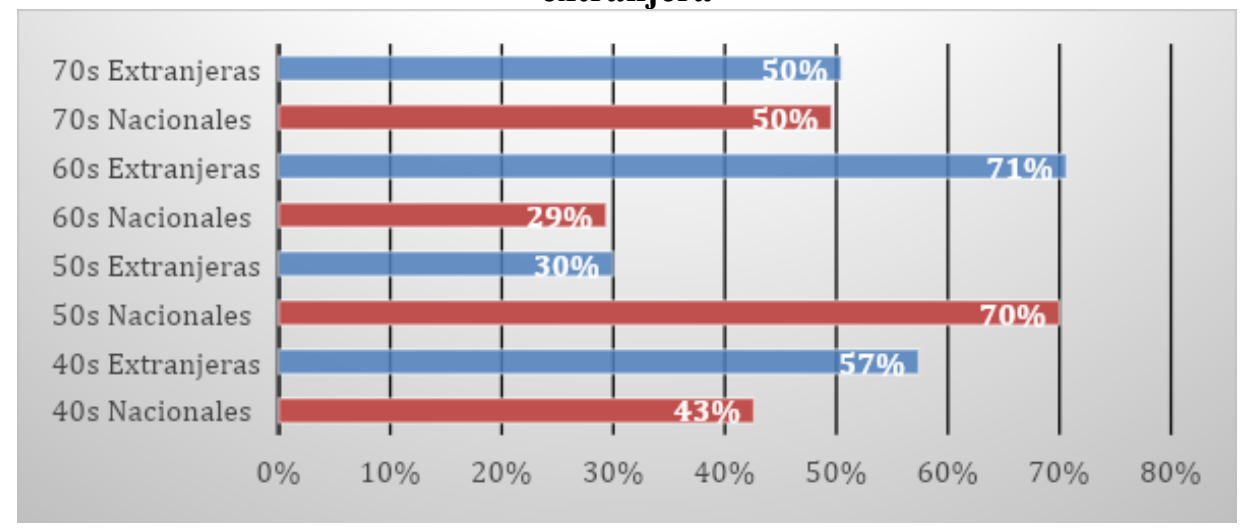

Fuente: Elaboración propia a partir de la estadística de programación en carteleras sabatinas de cine en León por cada década.

Tabla 3. Porcentajes de programación por origen de producción, nacional y extranjera, décadas cuarenta a setenta y distinción de cine para audiencias de clase popular

\begin{tabular}{|c|c|c|c|c|c|c|c|c|c|}
\hline Década & Origen & Isabel & Américas & Coliseo & León & Reforma & Estrella & Guanajuato & Total \\
\hline 40 & Nacionales & $3 \%$ & & $97 \%$ & & & & & $100 \%$ \\
\hline 40 & Extranjeras & & & & & & & & $0 \%$ \\
\hline 50 & Nacionales & $33 \%$ & & $43 \%$ & & & & & $76 \%$ \\
\hline 50 & Extranjeras & $18 \%$ & & $6 \%$ & & & & & $24 \%$ \\
\hline 60 & Nacionales & $23 \%$ & $3 \%$ & & $7 \%$ & $22 \%$ & & & $54 \%$ \\
\hline 60 & Extranjeras & $46 \%$ & & & & & & & $46 \%$ \\
\hline 70 & Nacionales & & $3 \%$ & $51 \%$ & $13 \%$ & $25 \%$ & $7 \%$ & $1 \%$ & $99 \%$ \\
\hline 70 & Extranjeras & & & & & & & $1 \%$ & $1 \%$ \\
\hline
\end{tabular}

Fuente: Elaboración propia. Los datos se refieren a la cartelera en 52 sábados por año.

Tabla 4. Porcentajes de programación por origen de producción, nacional y extranjera, décadas de los años cuarenta a setenta, y distinción de cine para audiencias de clase acomodada

\begin{tabular}{|c|c|c|c|c|c|c|c|c|}
\hline Década & Origen & Hernán & Ideal & $\begin{array}{c}\text { Teatro } \\
\text { Doblado }\end{array}$ & Vera & Insurgentes & $\begin{array}{c}\text { Autocinema } \\
\text { Valle }\end{array}$ & Total \\
\hline 40 & Nacionales & $8 \%$ & $16 \%$ & $8 \%$ & $8 \%$ & & & $40 \%$ \\
\hline 40 & Extranjeras & $60 \%$ & & & & & & $60 \%$ \\
\hline 50 & Nacionales & $22 \%$ & & & $40 \%$ & & & $63 \%$ \\
\hline 50 & Extranjeras & $28 \%$ & & & $10 \%$ & & & $38 \%$ \\
\hline 60 & Nacionales & $26 \%$ & & & & & & $26 \%$ \\
\hline 60 & Extranjeras & $74 \%$ & & & & & & $74 \%$ \\
\hline 70 & Nacionales & $25 \%$ & & & $47 \%$ & $5 \%$ & $1 \%$ & $78 \%$ \\
\hline 70 & Extranjeras & $22 \%$ & & & & & & $22 \%$ \\
\hline
\end{tabular}

Fuente: Elaboración propia. Los datos se refieren a la cartelera en 52 sábados por año. 
Una premisa de trabajo para explicar el comportamiento de la proyección de películas tiene que atender a diferentes circunstancias contextuales. De manera general, la distinción en el consumo de cine de acuerdo al nivel socioeconómico de la audiencia es la pauta explicativa para las dos primeras décadas, mientras que, a partir de 1960 únicamente se trabaja con procesos estructurales vinculados al crecimiento urbano de la ciudad.

Figura 2. Porcentaje de programación según su origen de producción, nacional o extranjera, en cines con distinción de audiencias, acomodadas o populares. Décadas de los años cuarenta y cincuenta
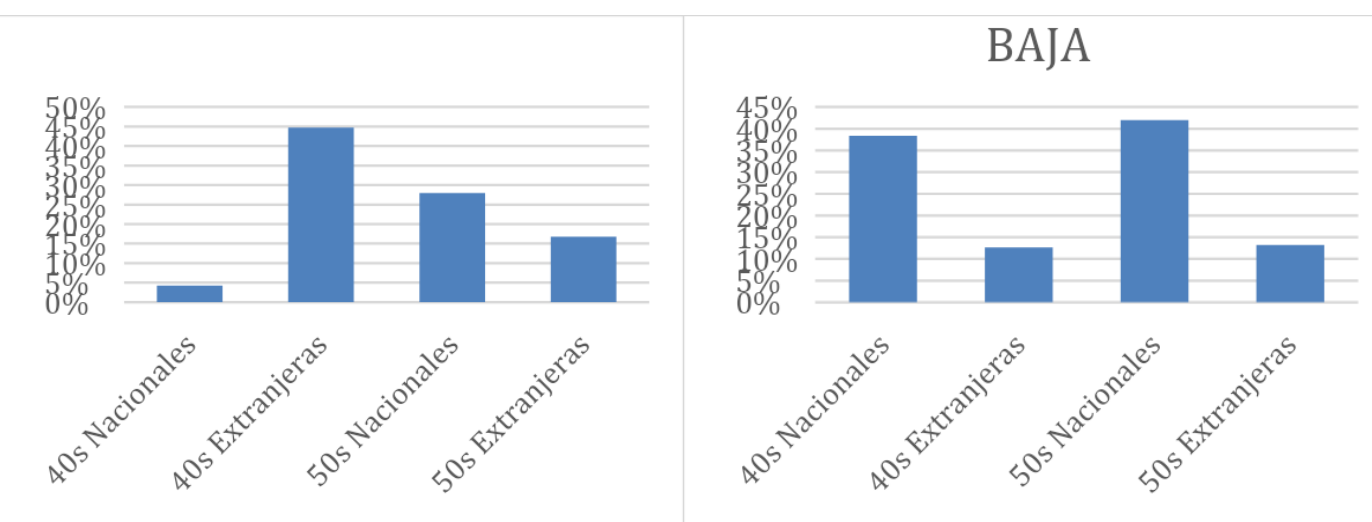

Fuente: Elaboración propia.

A partir de 1940, tanto las películas extranjero, de las cuales, $78 \%$ se proyectaban para salas con audiencias de de origen nacional como extranjero, ocupan cada una un público diferenciado. clase alta o acomodada. Por otro lado, el consumo de películas de origen nacional parecería estar supeditado principalmente a salas con proyecciones para la audiencia Cincuenta y ocho por ciento del total de popular, transmitiendo $90.5 \%$ de las proyecciones en la ciudad son de origen producciones totales, que representaban $38 \%$.

Tabla 5. Programación cinematográfica según origen, nacional o extranjera, con distinción de audiencias, acomodadas o populares. Programación década de 1940

\begin{tabular}{lcc}
\hline & Acomodadas & Populares \\
\hline 40s Nacionales & $4 \%$ & $38 \%$ \\
40s Extranjeras & $45 \%$ & $13 \%$ \\
\hline
\end{tabular}

Fuente: Elaboración propia. 
De este periodo son películas como Regalo de Reyes, con Sara García; Allá en el bajío, con Pedro Armendáriz; o, Cuando viajan las estrellas, con participación de Jorge Negrete y Gabriel Figueroa.

La década de los años cincuenta muestra un incremento exponencial de programación de cine mexicano, particularmente en salas con audiencias de niveles económicos altos, alcanzando $28 \%$ de participación. Este periodo es correspondiente con la segunda década del llamado Cine de Oro Mexicano, en el cual existe históricamente una disminución de la calidad en las producciones mexicanas, mermadas por el repunte de la producción norteamericana, impuestos a las exportaciones y el abandono de las políticas de apoyo a la producción mexicana al finalizar la segunda guerra mundial (Fein,
1994). No obstante, contrario a lo que pasó en otras ciudades, como México y Monterrey, donde la oferta de salas comienza a contraerse en la década de 1950 (Mantecón, 2000; Lozano et al., 2012), en León se amplió la oferta de salas justamente a partir de los cincuenta hasta alcanzar su auge en los setenta. Se conjuga la carencia de oferta en el terreno del ocio y el comienzo del boom económico, que respecto a otras urbes siempre ha llevado un desfase de casi una década.

En general, el consumo de la ciudad en esta década fue de $70 \%$ de cine nacional contra un $30 \%$ de cine extranjero. Estados Unidos (23\%) y España (3\%) son los principales productores foráneos; Reino Unido, Italia, Cuba y Argentina se reparten el $4 \%$ de las producciones restantes.

Tabla 6. Programación cinematográfica según origen, nacional o extranjera, con distinción de audiencias, acomodadas o populares. Programación década de 1950

\begin{tabular}{lcc}
\hline & Acomodadas & Populares \\
\hline 50s Nacionales & $28 \%$ & $42 \%$ \\
50s Extranjeras & $17 \%$ & $13 \%$ \\
\hline
\end{tabular}

Fuente: Elaboración propia

A partir de 1960 se incorporan nuevos cines en la ciudad, como los cines Reforma y Américas, el Cine León, Cine Estrella o el Insurgentes. En esta década, el cine mexicano deja de ser un referente y se produce el declive del consumo de producciones nacionales.

En León sucede una total reinversión del consumo, contrario a la década pasada. El cine nacional tiene una baja sustancial, pasando de proyectarse del 70 a un $29 \%$, mientras que el cine extranjero presenta un amplio incremento.

De estos años se pueden recordar algunas películas proyectadas como $E l$ ángel exterminador (1962), de Luis Buñuel, protagonizada por Silvia Pinal, Enrique Rambal, Claudio Brook y 
Jacqueline Andere; Macario (1960); Los Caifanes (1967); Hasta el viento tiene miedo (1968); El libro de piedra (1969) entre otras.

En los años setenta, en un afán por reducir la influencia estadounidense en el país, el Estado mexicano se hace del control de la producción cinematográfica. El presidente Luis Echeverría (1970-1976) coloca a su hermano, Rodolfo Echeverría, como director del Banco Nacional Cinematográfico, el cual se encargaría de

financiar la producción y distribución de cine mexicano.

Resultado del apoyo al cine independiente, se abren las salas a nuevas temáticas con tintes sociales, políticos y sexuales. Esta época, también nombrada "el nuevo cine mexicano", es considerada uno de los momentos más importantes del cine nacional. En los primeros seis años de la década, se producen 532 películas. En León este impulso se observa en un repunte del consumo de cine nacional, con un aumento de hasta $21 \%$.

\section{Conclusiones}

El estudio de caso de la exhibición y programación cinematográfica en León de las décadas de 1940 a 1970, desde la perspectiva de New Cinema History, se suma a una serie de trabajos aplicados en distintas ciudades del mundo que permiten compartir información a partir de metodologías de investigación comparable.
$\mathrm{Si}$ el cine constituye una fuente modernizadora de la vida en la ciudad de León no puede responderse aún desde esta exploración de la exhibición y programación cinematográfica. Hace falta profundizar en la estructura de distribución de las cadenas operadoras y agentes de cada época - lo cual está en proceso-. A diferencia del trabajo en otras ciudades, la ciudad de León carece de un registro documental suficiente, por lo que los datos aportados en este trabajo representan un avance significativo, a la vez que se promueve la discusión con otros proyectos realizados en ciudades industriales de diferentes regiones.

León ya se encontraba, en coincidencia con otras ciudades de provincia, en un proceso de modernización impulsado por el desarrollo económico nacional que fomentó la rápida transformación de zona rural a urbana.

En general, el consumo de cine en la ciudad se ve supeditado a las políticas públicas nacionales, las relaciones mercantiles de los dueños de las salas y su relación con los distribuidores. Sin embargo, la producción cultural cinematográfica nacional compite por posicionarse frente a las ofertas de películas extranjeras, especialmente estadounidenses. Esta pugna por constituirse como una industria de la cultura nacional se refleja en el consumo de la audiencia leonesa, aparentemente de manera caótica. 
Al analizar estadísticamente los programas de exhibición de estas décadas y las salas que ofertaron este servicio en León, se observa una adaptación de las carteleras a sus distintos públicos: los de clases populares consumían más cine nacional que las audiencias de clase acomodada.

Esta diferenciación es particularmente observable en la década de 1940, en la que, a la par de un creciente proceso de urbanización, se ostenta una brecha económica que se manifiesta tanto estructuralmente como en las prácticas de consumo del cine.

De la misma manera que sucedía en otras ciudades de provincia, poniendo el caso de Monterrey, las clases populares tenían “experiencias sociales muy diferentes a las experimentadas por sus contrapartes de clase media y alta que sólo acudían a los palacios ubicados en el centro de la ciudad" (Lozano et al., 2012, p. 87). Así, es en los años cuarenta y cincuenta donde existe un impulso importante en la programación de cine nacional como resultado de políticas públicas y económicas acordes a las necesidades de la industria cinematográfica, difundiendo contenidos más cercanos a la cultura local.

Durante los años sesenta, la producción de cine nacional presenta una baja relacionada principalmente con las estructuras económicas, según argumenta Paxman (2008) respecto al cine de oro mexicano. Una excepción es en la década de los setentas, en la que se impulsó desde el Gobierno federal la producción $y$ distribución del cine nacional para generar un repunte en la programación.

El análisis de la exhibición y la programación cinematográfica en León, representa un acercamiento empírico, que estaba pendiente, a la oferta y consumo de cine por su origen en el centro del país. Un abordaje necesario para complementar los estudios sobre la memoria social del cine, sus apropiaciones y las prácticas de consumo que con base en un enfoque sociocultural se realizan desde la perspectiva del New Cinema History.

\section{Referencias bibliográficas}

Alegre, L. (1995, otoño). El cine en León, los primeros años. Revista Andanzas, (10).

Allen, R. C. (2006). Relocating American film history. Cultural Studies, 20(1), 48-88.

Archivo Histórico de la Ciudad de León. (1998). Así era León. Ruel S.A. de C.V. Recuperado de https://www.ruelsa.com/gto/leon/leon4.htm

Biltereyst, D. (2007). De disciplinering van een medium. Filmvertoningen tijdens het Interbellum. En D. Biltereyst y P. Meers (eds.) De Verlichte Stad. Een geschiedenis van bioscopen, filmvertoningen en filmcultuur in Vlaanderen (pp. 45-61). Leuven: LannooCampus. 
Biltereyst, D. y Meers, P. (Eds.). (2007). De Verlichte Stad. Een geschiedenis van bioscopen, filmvertoningen en filmcultuur in Vlaanderen. Leuven: LannooCampus.

Biltereyst, D. y Meers, P. (2016). New cinema history and the comparative mode: Reflections on comparing historical cinema cultures. Alphaville: Journal of Film and Screen Media Issue, 11, 13-32.

Cortés Gaviño, A. (1995, otoño). Ensayo neurótico clasificado en C3. Revista Andanzas, (10).

Fein, S. (1994). Hollywood, U. S.-Mexican relations, and the devolution of the "Golden Age" of Mexican cinema. Film-Historia, IV(2), 103-135.

García Gómez, M. Á. (2010). Transformaciones urbanas de León siglo XX. León, México: Tlacuilo.

García Saavedra, J. (1985). Apuntes geográficos y estadísticos de la ciudad de León. En León en 1772. Edición facsimilar. León, México: Minerva Pleath.

González Chávez, J. M. (2016). Tres dimensiones de un despojo. Análisis antroponómico de la oferta educativa en León [Tesis doctoral]. Universidad Autónoma de Coahuila y Universidad Nacional Autónoma de México, México.

Guzmán López, M. Á. (2014). Guanajuato entre 1938 y 1949: Una década de crisis y cambio económico. Revista Oficio de Historia e Interdisciplina, 2(1), 115-141.

Instituto Municipal de Planeación Urbana. (2016). Polígonos de Pobreza de León. Recuperado en julio de 2016. http://www.implan.gob.mx

Instituto Nacional de Estadística Geografía e Historia. (2001). Indicadores sociodemográficos de México (1930-2000). Recuperado de http://internet.contenidos.inegi.org.mx/contenidos/productos/prod_serv/contenidos/es panol/bvinegi/productos/historicos/2104/702825493875/702825493875_1.pdf

Labarthe, M. de la C. (1995, otoño). Naranja dulce, limón partido. Los leoneses y su tiempo libre antes del cine. Revista Andanzas (10). "Cien años de Cine en León. Ese obscuro objeto del deseo".

Leal, J. F. y Barra, E. (2017). Anales del cine vol. 12 en México 1895-1911 1906: los cines pueblan la ciudad de México. México: Voyeur / UNAM.

Lewis, O. (1958). México desde 1940. Investigación Económica, 18(70), 185-256.

Lozano, J. C., Biltereys, D., Frankenberg, L., Meers, P. e Hinojosa, L. (2012) Exhibición y programación cinematográfica en Monterrey, México de 1922 a 1962: un estudio de caso desde la perspectiva de la "nueva historia del cine". Global Media Journal. Volumen 9, Número 18 Pp. 73-94. México.

Lozano, J.C. (2017). Film at the border. Memories of cinema going in Laredo, Texas, 1930s1960s. Memory Studies, 10(1), 35-48.

Lozano, J.C., Biltereyst, D. y Meers, Ph. (2017) Naïve and sophisticated long-term readings of foreign and national films viewed in a Mexican Northern town during the 1930-60s. 
Studies in Spanish \& Latin American Cinemas, 14(3), 277-296. doi: https://doi.org/10.1386/slac.14.3.277_1

Maltby, R., Biltereyst, D. y Meers, P. (Eds.). (2011). Explorations in new cinema history: Approaches and case studies, United Kingdom, Blackwell Publishing Ltd.

Mantecón, A. R. (2000) Auge, ocaso y renacimiento de la exhibición de cine en la ciudad de México (1930-2000). Alteridades, 10(20): 107-116.

Meers, P., Biltereyst, D. y Van de Vijver, L. (2010). Metropolitan vs rural cinemagoing in Flanders, 1925-1975. Screen, 51(3), 272-280.

Meers, Ph., Biltereyst, D. y Van de Vijver, L. (2008). Lived experiences of the 'Enlightened City' (1925-1975). A large scale oral history project on cinema-going in Flanders (Belgium), Iluminace. Journal of Film Theory, History and Aesthetics, 20(1), 208-214.

Mosco, V. (2006) La Economía Política de la Comunicación: una actualización diez años después. CIC Cuadernos de Información y Comunicación, 11 (pp. 57-79). Madrid, España: Universidad Complutense de Madrid.

Paxman, A. (2008). William Jenkins, Business Elites, and the Evolution of the Mexican State: 1910-1960 [Tesis de doctorado]. University of Texas, Austin, Texas, Estados Unidos.

Repoll, J., Portillo, M. y Meers, P. (2014) ¿Qué hubiera sido de mi vida sin el cine? La experiencia cinematográfica en la Ciudad de México. Contratexto, (22): 213-228.

Scolari, Carlos (2008) Las Hipermediaciones. Elementos para una teoría de la comunicación digital interactiva. Barcelona, España: Gedisa. 